\title{
Modeling of particle size segregation: Calibration using the discrete particle method
}

Article in Neuroethics · January 2011

DOI: $10.1142 / 50129183112400141$

\section{CITATIONS}

70

4 authors, including:

\section{Anthony Thornton}

University of Twente

56 PUBLICATIONS 844 CITATIONS

SEE PROFILE

\section{Onno Bokhove}

University of Leeds

132 PUBLICATIONS 1,158 CITATIONS

SEE PROFILE
Thomas Weinhart

University of Twente

49 PUBLICATIONS 564 CITATIONS

SEE PROFILE

Some of the authors of this publication are also working on these related projects:

Project SurfsUp View project

Geometric Dynamics View project 


\title{
MODELING OF PARTICLE SIZE SEGREGATION: CALIBRATION USING THE DISCRETE PARTICLE METHOD
}

\author{
ANTHONY THORNTON*,†,*, THOMAS WEINHART ${ }^{*, \dagger}$, \\ STEFAN LUDING* and ONNO BOKHOVE ${ }^{\dagger}$ \\ *Department of Mechanical Engineering: Multi-Scale Mechanics \\ University of Twente, P. O. Box 217, 7500 AE Enschede, The Netherlands \\ ${ }^{\dagger}$ Department of Mathematics: Mathematical Analysis and Computational Science \\ University of Twente, P. O. Box 217, 7500 AE Enschede, The Netherlands \\ \$a.r.thornton@utwente.nl
}

Received 20 October 2011

Accepted 30 January 2012

Published 15 August 2012

\begin{abstract}
Over the last 25 years a lot of work has been undertaken on constructing continuum models for segregation of particles of different sizes. We focus on one model that is designed to predict segregation and remixing of two differently sized particle species. This model contains two dimensionless parameters: $S_{r}$, a measure of the segregation rate, and $D_{r}$, a measure of the strength of diffusion. These, in general, depend on both flow and particle properties and one of the weaknesses of the model is that these dependencies are not predicted. They have to be determined by either experiments or simulations.

We present steady-state, periodic, chute-flow simulations using the discrete particle method (DPM) for several bi-disperse systems with different size ratios. The aim is to determine one parameter in the continuum model, i.e. the segregation Péclet number (ratio of the segregation rate to diffusion, $S_{r} / D_{r}$ ) as a function of the particle size ratio.

Reasonable agreement is found; but, also measurable discrepancies are reported; mainly, in the simulations a thick pure phase of large particles is formed at the top of the flow. Additionally, it was found that the Péclet number increases linearly with the size ratio for low values, but saturates to a value of approximately 7.7 .
\end{abstract}

Keywords: Granular materials; DPM (DEM); segregation; continuum approach.

PACS Nos.: 81.05.Rm, 45.70.Mg.

\section{Introduction}

Granular materials are common in industry and nature; in both situations segregation plays an important, but poorly understood, role in the flow dynamics. ${ }^{1,2}$ There are many mechanisms for the segregation of dissimilar grains in granular flows; however, segregation due to size-differences is often the most important. ${ }^{3}$ This study 


\section{A. Thornton et al.}

will focus on dense granular chute flows where kinetic sieving ${ }^{4,5}$ is the dominant mechanism for particle-size segregation. The basic idea is: As the grains avalanche down-slope, the local void ratio fluctuates and small particles preferentially fall into the gaps that open up beneath them, as they are more likely to fit into the available space than the large ones. The small particles, therefore, migrate towards the bottom of the flow and lever the large particles upwards due to force imbalances. This was termed squeeze expulsion by Savage and Lun. ${ }^{5}$

In this paper we will use the discrete particle method (DPM), ${ }^{6,7}$ also known as the discrete element method, to investigate segregation in dense granular chute flows. The ultimate aim is to use DPM both to validate the assumptions of kinetic sieving-based segregation models, and to aid with the calibration of the free parameters that appear in these models. Despite the large number of DPM studies of segregation in industrial chutes, and other apparatus, very few systematic studies have been performed for straightforward chute flows. Using simple chute geometries allows the results to be more easily compared to continuum theories enabling the determination of macro-parameters as a function of the DPM micro-parameters. We take a step in this direction by investigating how the ratio of the strength of segregation to diffusion depends on the size ratio.

\section{The Discrete Particle Method}

Many researchers have used DPM simulations to investigate segregation in a huge variety of situations and in this short paper it will not be possible to review them. However, of particular interest to this research is the investigation of steady-state segregation profiles for varying density and size differences in chute flows by Khakhar et al. ${ }^{8}$ They used DPM to investigate density effects and Monte Carlo simulations for different-sized particles. ${ }^{\mathrm{a}}$

We perform simulations of a collection of bi-dispersed spherical granular particles of density $\rho_{p}$. Each particle $i$ has a position $\boldsymbol{r}_{i}$, diameter $d_{i}$ and velocity $\boldsymbol{v}_{i}$. The relative distance is $r_{i j}=\left|\boldsymbol{r}_{i}-\boldsymbol{r}_{j}\right|$, and two particles are in contact if their overlap, $\delta_{i j}^{n}=\max \left(0,\left(d_{i}+d_{j}\right) / 2-r_{i j}\right)$, is positive. For interactions the normal, $\boldsymbol{f}_{i j}^{n}$, and tangential, $\boldsymbol{f}_{i j}^{t}$, forces are modeled as a spring-dashpot ${ }^{6,7}$ with linear elastic and linear dissipative contributions, with spring constants $k^{n}, k^{t}$ and damping coefficients $\gamma^{n}$, $\gamma^{t}$. When the tangential-to-normal force ratio becomes larger than a contact friction coefficient, $\mu^{c}$, the tangential spring yields and the particles slide, and we truncate the magnitude of the tangential spring as necessary to satisfy $\left|\boldsymbol{f}_{i j}^{t}\right| \leq \mu^{c}\left|\boldsymbol{f}_{i j}^{n}\right|$. For more details on the contact law used in these simulations we refer the reader to Weinhart et al. $;^{7}$ whereas, in Luding ${ }^{6}$ a more complete review of contact laws, in general, can be found. The base was created by adding fixed small particles, of

a After the initial submission, we became aware of the recent study by Tripathi and Khakhar. ${ }^{9}$ They performed a very detailed investigation of the rheology of binary-species flows differing in both size and density, which is relevant to this work. 
diameter $d_{s}=0.6 \mathrm{~mm}$, randomly, to a flat surface. The fixed small particles are endowed with an infinite mass and thus do not move.

Simulation parameters were chosen based on particles with $\rho_{p}=2400 \mathrm{~kg} \mathrm{~m}^{-3}$, $g=9.81 \mathrm{~ms}^{-1}$ and a small particle diameter of $d_{s}=0.6 \mathrm{~mm}$. The parameters of the normal forces were taken to be $k^{n}=29.00 \mathrm{Nm}^{-1}$ and $\gamma^{n}=0.0017 \mathrm{~kg} \mathrm{~s}^{-1}$ such that the restitution coefficient, for a collision between two small particles, is $r_{c}=0.6$ and the collision time $t_{c}=0.005 \sqrt{d_{s} / g}$ for the small particles, where $\sqrt{d_{s} / g}$ is the small particle diameter to gravitational acceleration timescale. In total, 10 simulations were performed for values of the diameter ratio, $\sigma^{-1}=d_{l} / d_{s}$, from 1.1 to 2.0 in steps of 0.1. Similarly, parameters for the tangential forces are given by $k^{t}=(2 / 7) k^{n}$, $\gamma^{t}=\gamma^{n}$ and $\mu^{c}=0.8$.

Simulations using only normal forces in the contact model were also undertaken, but these flows did not settle to a stable steady state. Also, with only normal forces the flow occasionally spontaneously compacted, reducing its thickness by around $15 \%$ and then slowly dilated back to approximately its original density. To the best of our knowledge this effect has not been observed in experiments and, hence, we disregarded these results from our analysis.

Obtaining macroscopic fields from DPM simulations is a nontrivial task, especially near a boundary. Here, we use the spatial coarse-graining procedure that is still valid near a boundary described by Weinhart et al. ${ }^{10} \mathrm{We}$ only require the volume of the small particles,

$$
V^{s}(\boldsymbol{r}, t)=\frac{\pi}{6} d_{s}^{3} \sum_{i \in \mathcal{S}} \mathcal{W}\left(\boldsymbol{r}-\boldsymbol{r}_{i}(t)\right),
$$

the total particle volume,

$$
V(\boldsymbol{r}, t)=\frac{\pi}{6} \sum_{i} d_{i}^{3} \mathcal{W}\left(\boldsymbol{r}-\boldsymbol{r}_{i}(t)\right)
$$

and, downwards normal stress $\sigma_{z z}$ (height); where $\mathcal{W}$ is a coarse-graining function, and $\mathcal{S}$ denotes the set of small particles. In this paper, $\mathcal{W}$ is taken to be a Gaussian of width, or variance, $d_{s} / 2$.

\section{Continuum Model of Segregation}

The first model of kinetic sieving was developed by Savage and Lun, ${ }^{5}$ using a statistical argument about the distribution of void spaces. Later, Gray and Thornton ${ }^{11}$ developed a similar model from a mixture-theory framework. Their derivation has two key assumptions: Firstly, as the different particles percolate past each other there is a Darcy-style drag between the different constituents (i.e. the small and large particles) and, secondly, particles falling into void spaces do not support any of the bed weight. Since the number of voids available for small particles to fall into is greater than for large particles, it follows that a higher percentage of the small particles will be falling and, hence, not supporting any of the bed load. In recent 
years, this segregation theory has been developed and extended in many directions: Including the addition of a passive background fluid, ${ }^{12}$ the effect of diffusive remixing, ${ }^{13}$ and the generalization to multi-component granular flows. ${ }^{14}$ We will use the two-particle size segregation-remixing version derived by Gray and Chugunov; ${ }^{13}$ however, it should be noted that Dolgunin and Ukolov ${ }^{15}$ were the first to suggest this form, by using phenomenological arguments. The bi-dispersed segregation-remixing model contains two dimensionless parameters. These in general will depend on flow and particle properties, such as: size-ratio, material properties, shear-rate, slope angle, particle roughness, etc. One of the weaknesses of the model is that it is not able to predict the dependence of the two parameters on the particle and flow properties. These have to be determined by either experiments or DPM simulations.

The two-particle segregation-remixing equation ${ }^{13}$ takes the form of a non-dimensional scalar conservation law for the small particle concentration $\phi$ as a function of the spatial coordinates $\hat{x}, \hat{y}$ and $\hat{z}$; and, time $\hat{t}$,

$$
\frac{\partial \phi}{\partial \hat{t}}+\frac{\partial}{\partial \hat{x}}(\phi \hat{u})+\frac{\partial}{\partial \hat{y}}(\phi \hat{v})+\frac{\partial}{\partial \hat{z}}(\phi \hat{w})-\frac{\partial}{\partial \hat{z}}\left(S_{r} \phi(1-\phi)\right)=\frac{\partial}{\partial \hat{z}}\left(D_{r} \frac{\partial \phi}{\partial \hat{z}}\right),
$$

where $S_{r}$ is the dimensionless measure of the segregation-rate, whose form in the most general case is discussed in Thornton, Gray and $\mathrm{Hogg}^{12}$ and $D_{r}$ is a dimensionless measure of the diffusive remixing. In (3), $\partial$ is used to indicate a partial derivative, and the "hat" a dimensionless variable; $\hat{x}$ is the down-slope coordinate, $\hat{y}$ the cross-slope coordinate and $\hat{z}$ the coordinate normal to the base. Furthermore $\hat{u}, \hat{v}$ and $\hat{w}$ are the dimensionless bulk velocity components in the $\hat{x}, \hat{y}$ and $\hat{z}$ directions, respectively. The conservation law (3) is derived under the assumption of uniform porosity and is often solved subject to the condition that there is no normal flux of particles through the base or free surface of the flow.

\subsection{Steady-state solution}

We limit our attention to small-scale DPM simulations, periodic in the $x$ - and $y$-directions, and investigate the final steady-states. Therefore, we are interested in a steady-state solution to (3) subject to no-normal flux boundary condition, at $\hat{z}=0$ (the bottom) and 1 (the top), that is independent of $\hat{x}$ and $\hat{y}$. Gray and Chugunov ${ }^{13}$ showed that such a solution takes the form,

$$
\phi(\hat{z})=\frac{\left(1-e^{\left(-\phi_{0} P_{s}\right)}\right) e^{\left(\phi_{0}-z\right) P_{s}}}{1-e^{\left(-\left(1-\phi_{0}\right) P_{s}\right)}+\left(1-e^{-\phi_{0} P_{s}}\right) e^{\left(\phi_{0}-z\right) P_{s}}},
$$

where $P_{s}=S_{r} / D_{r}$ is the segregation Péclet number and $\phi_{0}$ is the mean concentration of small particles. This solution represents a balance between the last two terms of (3) and is related to the logistic equation. In general, $P_{s}$ will be a function of the particle properties, and we will use DPM to investigate the dependence of $P_{s}$ on the particle size ratio $\sigma=d_{s} / d_{l}$. It should be noted that $\sigma$ has been defined such that it is consistent with the original theory of Savage and Lun; ${ }^{5}$ however, with this definition 
only values between 0 and 1 are possible. Therefore, we will present the results in terms of $\sigma^{-1}$ which ranges from 1 to infinity.

\subsection{Nondimensionalization of the DPM results}

The DPM results presented in Sec. 4 will be averaged in the periodic $x$ - and $y$-directions; therefore, we will obtain the total granular volume, $V$, and small particle volume, $V_{s}$, as a function of $z$ only. For comparison with the nondimensional analytical solution (4) the results will be nondimensionalized by the scaling

$$
\hat{z}=\frac{(z-b)}{(s-b)}, \quad \phi=\frac{V^{s}}{V},
$$

where $b$ is the location of the base of the flow; $s$ the location of the free surface; and, $V^{s}$ and $V$, are given by Eqs. (1) and (2), respectively. Hence, the mean concentration, $\phi_{0}$, of small particles is given by

$$
\phi_{0}=\frac{1}{s-b} \int_{b}^{s} \phi(\hat{z}) \mathrm{d} z=\int_{0}^{1} \phi(\hat{z}) \mathrm{d} \hat{z} .
$$

It is then possible to directly compare (4), obtained subject to the no-normal flux condition at $\hat{z}=0$ and 1 , with the steady-state volume fraction profiles, $\phi(\hat{z})$, obtained from the simulations. Therefore, $P_{s}$ can be determined via a nonlinear regression fit of $\phi(\hat{z})$ to $(4)$, i.e. $P_{s}$ is used as the single free fitting parameter.

The free surface of the flow is not clearly defined in a DPM simulation and here two different definitions will be considered. Weinhart et al. ${ }^{7}$ investigated how to consistently define the base and free-surface locations for flows over rough bottoms. Following their results, the height of the flow is defined to be the distance between the point where the downwards normal stress $\sigma_{z z}$ vanishes and where it reaches its maximum value. In order to avoid the effects of coarse graining, the linear bulk stress profile between $2 \%$ and $98 \%$ of its maximum is linearly extrapolated to define the base and surface locations.

In granular chute flows there is a layer of saltating particles towards the top of the flow, where the density decreases with height. This effect is confirmed in the simulations presented here, see Fig. 4. Gray and Chugunov ${ }^{13}$ suggested that their theory should not be fitted over the less dense region and defined the top of the flow as the point where the density starts to decrease. Therefore, we will also use

$$
s_{d}=0.98 \max \rho
$$

to define the surface between the dense and less dense regions, where $\rho$ is the density of the flow. The value 0.98 was chosen because the basal layer density fluctuates by approximately $\pm 1 \%$. An illustration of the demarcation between dense and less dense flow is shown in Fig. 4. We will use the notation $\hat{z}_{f}$ to indicate a $z$-coordinate scaled by the free-surface location and $\hat{z}_{d}$ as a $z$-coordinate scaled by the location of the dense basal layer, i.e. by (7). 


\section{Previous Comparison to the Theory}

The original theory of Gray and Thornton ${ }^{11}$ has been used to investigate sheardriven segregation in an annular Couette cell. ${ }^{16,17}$ In that case good agreement between model and theory was found in the initial phase, but at later times the segregation rate exponentially slowed down, which is not captured by the model. Additionally, an increase in the thickness of the sample as the particle profile evolved (Reynolds dilatancy) was observed; this effect is also not included in the model. The measured segregation rates were found to be nonmonotonic in particle size-ratio; however, very large size-ratios (up-to $\sigma^{-1}=4$ ) were considered, for which the kinetic sieving process is known to start breaking down. Savage and Lun $^{5}$ showed that percolation effects are evident for $\sigma^{-1}>2$ and stated that spontaneous percolation occurs for $\sigma^{-1}>6.464$, i.e. small particles can percolate through the matrix of larger particles simply as a result of gravity, even in the absence of any shear.

Recently, chute experiments have been performed with a binary mixture of spherical glass particles with size-ratio of $\sigma^{-1}=2$ down an incline of $29^{\circ} .^{18}$ In these experiments it was very difficult to produce steady flow conditions and a dependence of the Péclet number on other parameters was observed. For these flow conditions Péclet numbers in the range 11-19 were reported.

Marks and Einav ${ }^{19}$ used a cellular automata model to investigate segregation in granular chute flows. They found good agreement between the model and their simulations. For simple shear configurations they found sharp jumps in concentration as predicted by the original low-diffusion theory. ${ }^{11}$

Khakhar, McCarthy and Ottino ${ }^{8}$ performed a detailed investigation of segregation, by both size and density differences in granular chute flows. For equal density different size particles they used Monte Carlo techniques to obtain steady-state profiles. Gray and Chugunov ${ }^{13}$ fitted this data to the steady-state solution (4). They found that for inelastic particles with $\sigma^{-1}=1.11$, on an incline of $25^{\circ}$, a Péclet number of 4 matched the data best. It should be noted that they did not fit across the entire layer of the flow, but from the top of the dense avalanching layer. Gray and Chugunov $^{13}$ used an ad hoc position for the location of the dense avalanching layer; we, however, will use definition (7).

\section{Measured Segregation Rates}

Figure 1 shows a series of images from the DPM simulations for different times and values of $\sigma^{-1}$. The simulations take place in a box: periodic in $x$ and $y, 5 d_{s}$ wide and $83.3 d_{s}$ long, and inclined at an angle of $25^{\circ}$ to the horizontal. The simulations are performed with 5000 flowing small particles and the number of large particles is chosen such that the total volume of large and small particles is equal, i.e. $\phi_{0}=0.5$ (to within the volume of one large particle). Initial conditions are randomly distributed without checking for overlaps; this creates a good homogeneous distribution of particles, but it does mean there are a few initial overlaps. This causes a small 

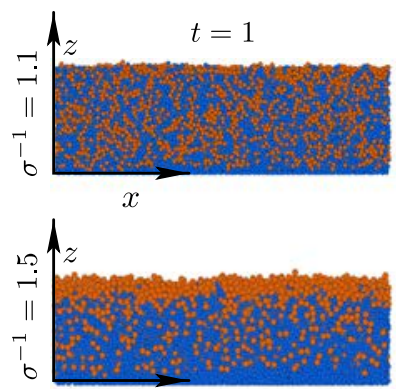

$x$

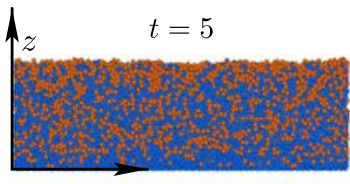

$x$

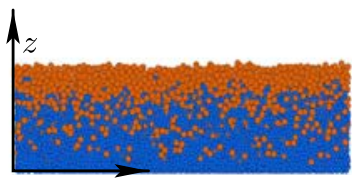

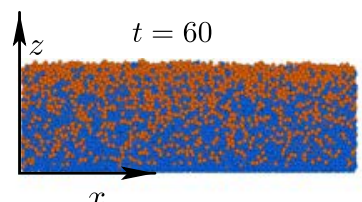

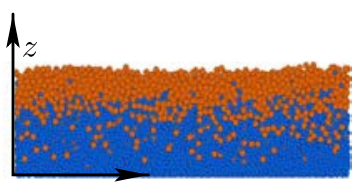

Fig. 1. (Color online) Snapshots from the DPM simulations with large (orange) and small (blue) particles. Rows correspond to distinct particle sizes and columns to different times. Top row $\sigma^{-1}=1.1$ and bottom row $\sigma^{-1}=1.5$; whereas, the left column is for $t=1$, middle $t=5$ and right $t=60$.

"explosion" of particles in the first few time steps, which is quickly dissipated and has no effect on the long-term evolution of the flow.

It can be seen from Fig. 1 that the larger $\sigma^{-1}$, the stronger the segregation. For the cases $\sigma^{-1}=1.3$ and higher a thick pure phase of large particles is formed at the top of the flow, but no equivalent thick pure small phase is formed at the base. At the base a very thin pure layer of small particles is formed which is at most two particle layers thick. This is due to the base comprising of small particles and, hence, only small particles can fit in the gaps in the basal surface. For $\sigma^{-1}=1.1$ some segregation can be observed; but, no pure layers are formed at either the top or the bottom.

To confirm the flow is in steady state, the vertical center-of-mass (COM) of the small and large particles is computed. Three examples are shown in Fig. 2. As can be seen, initially the COM of the small particles quickly decreases, while the COM of the large particles rises. This process slows down and eventually the COM becomes stable. For all the values of $\sigma^{-1}$ considered, the COM of the small particles reaches a constant value by $t=50$. To obtain good statistics about the $z$-dependence of $\phi$, the
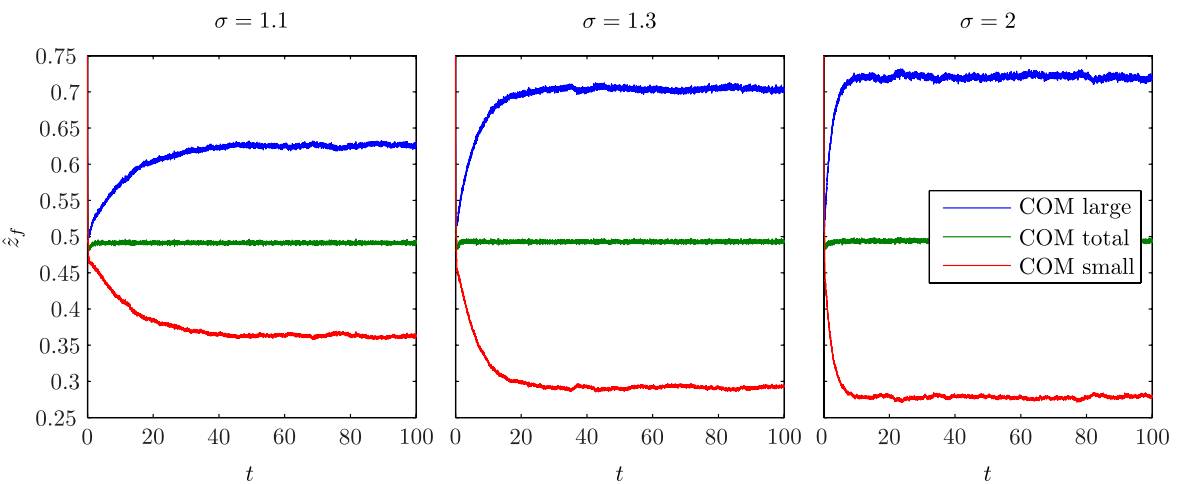

Fig. 2. (Color online) Center-of-mass (COM), scaled by the flow height, of the small particles (red), large particles (blue) and bulk (green) as a function of time. Plots for $\sigma^{-1}=1.1,1.3$ and $\sigma^{-1}=2$. 

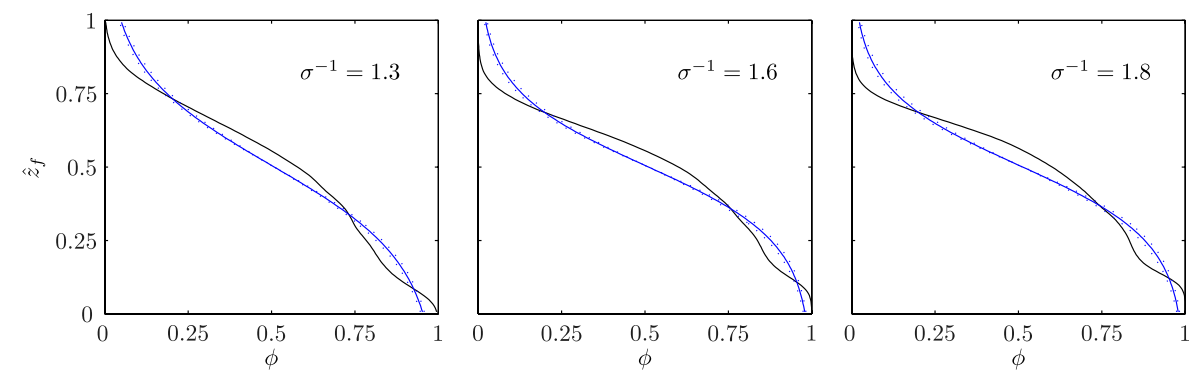

Fig. 3. (Color online) Plots of small particle volume fraction $\phi$ as a function of scaled depth $\hat{z}_{f}$. The black lines are the coarse-grained DPM simulation data and the blue lines are the fit to (4) produced with MATLAB's nonlinear regression function with dotted lines showing the $95 \%$ confidence intervals.

data will be averaged in both $x$ and $y$ over the interval $t \in[90,100]$; examples of these averaged coarse-grained depth profiles are presented in Fig. 3.

In Fig. 2, the bulk COM is also plotted. It remains roughly at the same depth while the segregation process is taking place. In the cases with larger size-ratio a change in the bulk COM can be seen between the homogeneous mixed initial conditions and the final segregated state. This is due to compaction effects present in mixed states, for large size ratios; but, currently this effect is not taken into account in the continuum model. A change in the flow thickness (COM) has also been observed in previous shear-driven size-segregation experiments. ${ }^{16,17}$

Additionally, from Fig. 2 it is clear that for smaller $\sigma^{-1}$, it takes longer for the flow to reach a steady state. This is an indication that the segregation rate $S_{r}$ is weaker for a smaller size difference, i.e. low $\sigma^{-1}$, and this lower $S_{r}$ could be the source of the lower Péclet number.

Figure 3 shows a fit of (4) to the small particle volume fraction for several cases. The fit is performed using nonlinear regression, as implemented in MATLAB, over the whole range depth of the flow, including the pure region of large particles on top. The fit is reasonable in all cases, especially considering there is only one degree of freedom, $P_{s}$. From these plots it is clear that the degree of segregation is stronger as the size ratio is increased. Note that, for $\sigma^{-1}>1.3$ a measurable pure phase of large particles is generated at the top of the flow, as also observed in Fig. 1, and this layer becomes thicker as the size-ratio is increased. Also, again, it can be seen that the flow becomes very rich in small particles at the base; but, only a thin pure phase is observed. Finally, in each case an inflection in the profile is observed towards the base, which is also not predicted by the theory; however, it was also observed in the Monte Carlo simulations of Khakhar, McCarthy and Ottino, ${ }^{8}$ replotted in terms of volume fraction by Gray and Chugunov. ${ }^{13}$

Gray and Chugunov ${ }^{13}$ have previously noted that their theory does not capture the perfectly pure region at the top of the flow and proposed only fitting to the dense basal layer. A typical volume fraction profile is shown in Fig. 4 and the decrease in density (bulk volume fraction) reported by Gray and Chugunov ${ }^{13}$ can be observed 


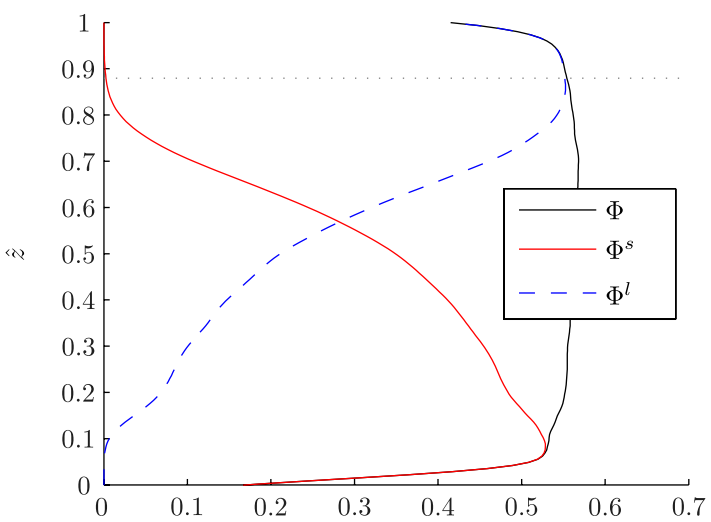

Fig. 4. (Color online) Plot of the volume fraction of both the small, $\Phi^{s}$ and large particles, $\Phi^{l}$ and the bulk solids volume fraction, $\Phi=\Phi^{s}+\Phi^{l}$, for the case $\sigma^{-1}=1.6$. The dotted line shows the demarcation line between the basal dense layer and the upper more dilute region as given by definition (7).

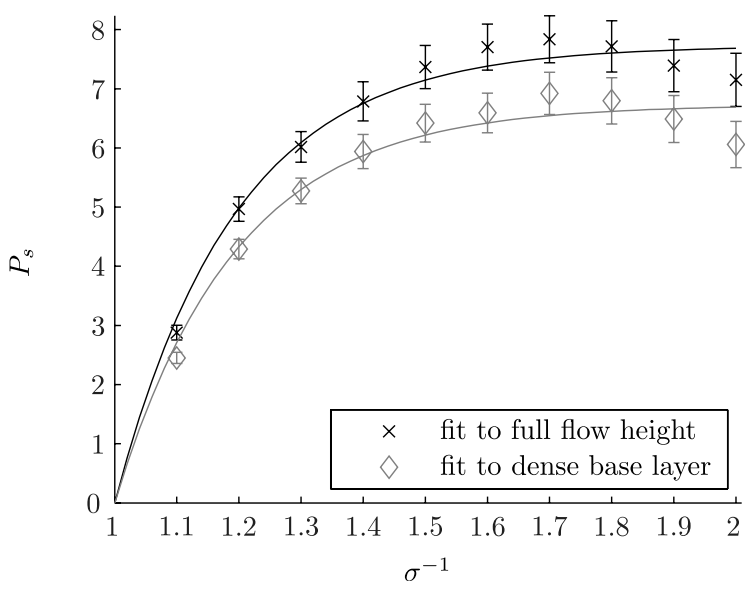

Fig. 5. Plot of the Péclet number, as obtained from the fit of (4) to the DPM data that are used as a function of particle-size ratio $\sigma^{-1}$. The crosses indicate data that is scaled using the full flow depth, i.e. $\hat{z}_{f}$; whereas, for the diamonds the flow is scaled by the thickness of the dense avalanche region, i.e. $\hat{z}_{d}$.

towards the top of the flow. Therefore, we use (7) to define the transition between the dense basal layer and the more dilute layer above (indicated by the dotted line in Fig. 4). Fits using this scaling were also performed. When scaling by the location of the basal layer a higher value of $\phi_{0}>0.5$ was computed, as these fits do not include the large particles in the upper part of the flow. The measured Péclet number for each type of fit is shown in Fig. 5 and from the error bars ( $95 \%$ confidence bounds) it can be seen that both fits have approximately the same accuracy. Therefore, nothing is gained by only fitting to the dense basal layer, and the free surface seems better defined. Hence, we will only consider scaling by the free surface location from this point onwards. 


\section{A. Thornton et al.}

Figure 5 shows the segregation Péclet number, $P_{s}$, as a function of $\sigma^{-1}$. Even for the smallest size ratio, $\sigma^{-1}=1.1, P_{s}$ is 2.9 , indicating that segregation is almost three times stronger than diffusion. For $\sigma^{-1}$ between 1.1 and 1.5 it would appear that $P_{s}$ saturates exponentially to a constant value of around $P_{\max }=7.73$. A fit is shown to $P_{s}=P_{\max }\left(1-e^{-k\left(\sigma^{-1}-1\right)}\right)$ where $k=5.18$ is the saturation constant. Further investigation is required on how $P_{s}$ depends on other parameters that appear in the contact model. Previously, Gray and Chugunov ${ }^{13}$ compared the model to a Monte Carlo result of Khakhar, McCarthy and Ottino, ${ }^{8}$ in which $\sigma^{-1}=1.11$, and found that a value of $P_{s}=4$ fitted the data best. This is a little larger than the value reported here, but the numerical model and particle properties are different between ours and Khakhar et al. simulations. Previous chute experiments ${ }^{18}$ reported a range of Péclet numbers from $11-19$ for $\sigma^{-1}=2$, which again is larger than the values we find here, but these experiments were performed at a higher inclination angle.

For very large size ratios it does appear that the Péclet number is beginning to decrease, as reported by Golick and Daniels; ${ }^{16}$ however, the reduction is only slightly larger than the fitting error and therefore is not conclusive. We did not consider higher size ratios as percolation effects are not included in the Gray and Chugunov model; Savage and $\mathrm{Lun}^{5}$ report percolation has to be taken into account for values of $\sigma^{-1}>2$. Therefore, the model considered may be of limited applicability above this size ratio and more work is required in this area.

\section{Conclusions}

We presented a DPM study of how the ratio of particle size-segregation strength to diffusion, as defined in the Péclet number, $P_{s}$, depends on the size ratio of the particles. Previously, only a fit to a single simulation had been reported. ${ }^{13} \mathrm{We}$ compared DPM simulations with theoretical predictions for steady-states, to a previous Monte Carlo simulation and to experiments.

We showed that $P_{s}$ does increase for larger particle size ratios $\sigma^{-1}$, but appears to saturate to a constant value. The results of the DPM were compared to the binary segregation-remixing model of Gray and Chugunov. ${ }^{13}$ The agreement was reasonable, given that the model has only one free fitting parameter, $P_{s}$. The major difference between the model and DPM is the slight asymmetry in the segregation, with a thick pure phase of large particles forming at the top; but, only a thin perfectly pure layer of small particles appearing at the base of the flow. The weaker segregation found at the base has previously been observed and this effect could be captured in the model, by introducing diffusion that is a function of the fluctuation energy of the flow. It is known that the fluctuation energy is stronger towards the base and almost zero at the free surface. ${ }^{20}$ Additionally, a small change in the bulk center-of-mass was observed between homogeneous and segregated states; again this effect is not included in the continuum model. However, it could be incorporated into the threephase version of Thornton, Gray and Hogg ${ }^{12}$ as this explicitly models the air phase and, hence, can be extended to allow the bulk granular volume fraction to vary in 
height and evolve with time. In the future we aim to use the results of DPM simulations to improve the continuum models.

Further investigations are required to determine how the degree of segregation depends on the parameters of the DPM. Since only steady-state profiles were considered, it was only possible to ascertain the ratio of the strength of the diffusion to the strength of the segregation. To measure these two effects independently, timeevolving profiles must be considered, which requires a better procedure to produce the initial configurations.

We have taken the contact model properties of the large and small particles to be the same, i.e. $k^{n}, \gamma^{n}, k^{t}, \gamma^{t}$ and $\mu$; however, to gain closer agreement with the experiments it may be better to assume the material properties are equal for both particle types, i.e. bulk modulus, coefficient of restitution, and hence the contact properties become size dependent. Finally, we have shown that DPM can be used to check and validate the assumptions of continuum segregation models.

\section{Acknowledgments}

The authors would like to thank the Institute of Mechanics, Processes and Control, Twente (IMPACT) for its financial support. The DPM simulations performed for this paper are undertaken in Mercury-DPM, which was initially developed within this IMPACT program. It is primarily developed by A. R. Thornton, T. Weinhart and D. Krijgsman as a joint project between the Multi-Scale Mechanics (Mechanical Engineering) and the Mathematical Analysis and Computational Science (Applied Mathematics) groups at the University of Twente. The research presented will benefit our project "Polydispersed Granular Flows through Inclined Channels" funded by STW.

\section{References}

1. R. M. Iverson, in Debris-Flow Hazards Havards Mitigation: Mechanics, Prediction and Assessment (Millpress, 2003), pp. 303-314.

2. T. Shinbrot, A. Alexander and F. J. Muzzio, Nature 397, 675 (1999).

3. J. A. Drahun and J. Bridgewater, Powder Technol. 36, 39 (1983).

4. G. V. Middleton, Flysch Sedimentology in North America, ed. J. Lajoie (Business and Economics Science Ltd, Toronto, 1970), pp. 253-272.

5. S. B. Savage and C. K. K. Lun, J. Fluid Mech. 189, 311 (1988).

6. S. Luding, Granul. Matter 10, 235 (2008).

7. T. Weinhart, A. R. Thornton, S. Luding and O. Bokhove, Granul. Matter 14, 531 (2012).

8. D. V. Khakhar, J. J. McCarthy and J. M. Ottino, Chaos 9, 594 (1999).

9. A. Tripathi and D. V. Khakhar, Phys. Fluids, in press, DOI: 10.1063/1.3653276.

10. T. Weinhart, A. R. Thornton, S. Luding and O. Bokhove, Granul. Matter 14, 289 (2012).

11. J. M. N. T. Gray and A. R. Thornton. Proc. Roy. Soc. A 461, 1447 (2005).

12. A. R. Thornton, J. M. N. T. Gray and A. J. Hogg. J. Fluid Mech. 550, 1 (2006).

13. J. M. N. T. Gray and V. A. Chugunov, J. Fluid Mech. 569, 365 (2006).

14. J. M. N. T. Gray and C. Ancey, J. Fluid Mech. 678, 535 (2011).

15. V. N. Dolgunin and A. A. Ukolov, Powder Tech. 83, 95 (1995). 
16. L. A. Golick and K. E. Daniels, Phys. Rev. E 80, 042301 (2009).

17. L. B. H. May, L. A. Golick, K. C. Phillips, M. Shearer and K. E. Daniels, Phys. Rev. E 81, 051301 (2010).

18. S. Weiderseiner, N. Andreini, G. Epely-Chauvin, G. Moser, M. Monnereau, J. M. N. T Gray and C. Ancey, Phys. Fluids 23, 013301 (2011).

19. B. Marks and I. Einav, Granul. Matter 13, 211 (2011).

20. Y. Forterre and O. Pouliquen, J. Fluid Mech. 467, 361 (2002). 